\section{Frontal Eye Fields}

Chava Creque ${ }^{1}$ and

Stephanie A. Kolakowsky-Hayner ${ }^{2}$

${ }^{1}$ Brain Trauma Foundation, New York, NY, USA

${ }^{2}$ Stanford Concussion and Brain Performance

Center, Palo Alto, CA, USA

\section{Synonyms}

Eye fields

\section{Definition}

The frontal eye fields (FEF) are part of the prefrontal cortex and considered to be Brodmann area 8 and portions of area 9 . The FEF receive input from numerous brain regions and seem to coordinate and maintain eye and head movements and gaze shifts and are involved in the orientation and attention responses to stimuli. Overall, the
FEF area receives information from the auditory, tactual, and visual environment and is considered to be a multimodal response area.

\section{Cross-References}

- Eye Fields

- Saccadic Eye Movements

- Visual-Motor Function

- Visual Tracking

\section{References and Readings}

Crowne, D. (1983). The frontal eye field and attention. Psychological Bulletin, 93(2), 232-260.

Machado, L., \& Rafal, R. (2004). Control of fixation and saccades in humans with chronic lesions of oculomotor cortex. Neuropsychology, 18(1), 115-123.

Wong, B., Cronin-Golomb, A., \& Neargarder, S. (2005). Patterns of visual scanning as predictors of emotion identification in normal aging. Neuropsychology, 19(6), 739-749. 